Supporting Information

\title{
3D-Printed Photoactive Semiconducting Nanowire-Polymer Composites for Light Sensors
}

Xin Shan ${ }^{1}$, Pengsu Mao ${ }^{1}$, Haoran $\mathrm{Li}^{1}$, Thomas Geske², Divya Bahadur ${ }^{3}$, Yan Xin ${ }^{4}$, Subramanian

Ramakrishnan ${ }^{3}$, Zhibin $\mathrm{Yu}^{1,2 *}$

1. Department of Industrial and Manufacturing Engineering, High-Performance Materials Institute, FAMU-FSU College of Engineering, Florida State University

2. Materials Science and Engineering, Florida State University

3. Department of Chemical and Biomedical Engineering, FAMU-FSU College of Engineering, Florida State University

4. National High Magnetic Field Laboratory, Florida State University

*Email: zyu@,fsu.edu 
Table S1. Chemical structures of P3HT, thermal curable EPON resin and UV curable diacrylate

\begin{tabular}{|c|c|}
\hline Chemical Name & Chemical Structure \\
\hline P3HT & \\
\hline BPON Resin 862 & \\
\hline Bisphenol A ethoxylate diacrylate &
\end{tabular}

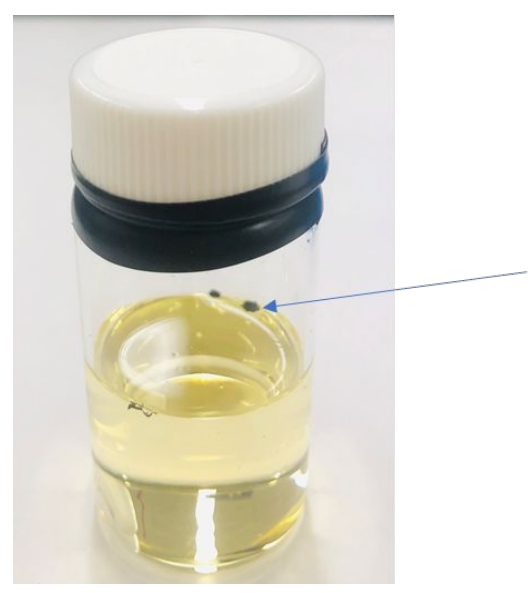

Undissolved P3HT

Figure S1. A photo of $1 \mathrm{mg}$ P3HT and $10 \mathrm{~mL}$ DCM mixture sealed in a glass vial. After stirring the mixture for 24 hours at room temperature, only a small amount of P3HT was dissolved in DCM. 


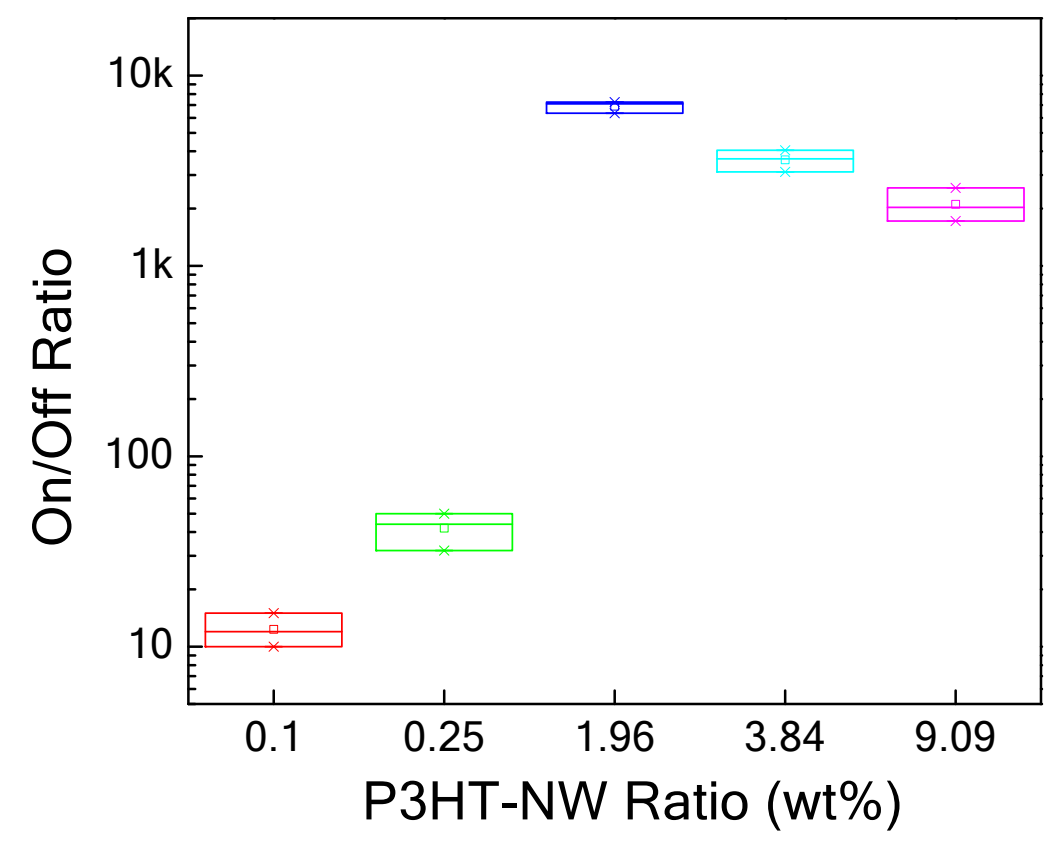

Figure S2. Statistic plots of On/Off ratios for devices with different P3HT nanowire concentrations. Three devices were prepared and measured from the same fabrication batch for each P3HT nanowire concentration. 

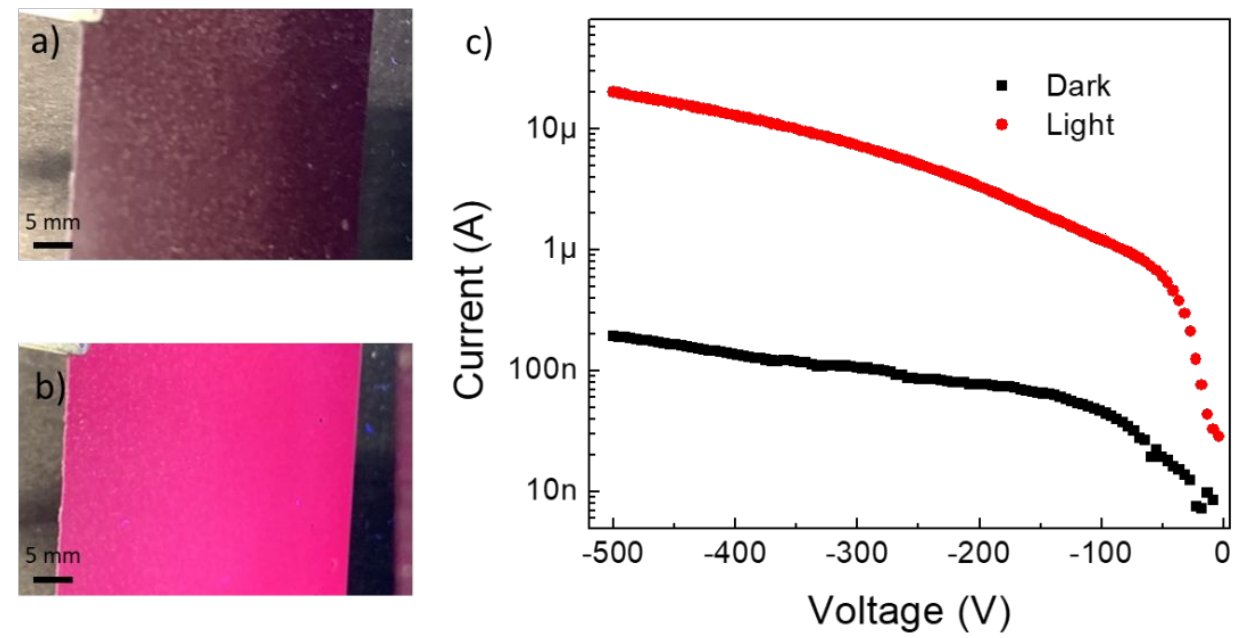

Figure S3. photos of a composite sheet with P3HT nanowire $(1.96 \mathrm{wt} \%)$ in a UV-cured acrylic polymer a) in ambient light condition and b) under $365 \mathrm{~nm}$ UV light. c) I-V characteristics from $500 \mathrm{~V}$ to $500 \mathrm{~V}$ of a photodetector using the P3HT nanowire (1.96 wt\%)-acrylic polymer composite. The composite was $100 \mu \mathrm{m}$ thick and the photodetector has a device structure as ITO/ P3HT nanowire (1.96 wt\%)-acrylic polymer composite/In-Ga. 